\title{
CXCL12 G801A polymorphism is associated with significant liver fibrosis in HIV-infected Thais: a cross-sectional study
}

\author{
Thitiilat Chiraunyanann, ${ }^{1,4}$ Khaimuk Changsri, ${ }^{2}$ Warisara Sretapunya, ${ }^{5}$ Kornanong Yuenyongchaiwat, ${ }^{3}$ Chareeporn Akekawatchai ${ }^{2}$
}

\begin{abstract}
Background: Previous studies indicate high prevalence of liver diseases in HIV-infected patients, and their genetic risk factors are still unclear. The chemokine CXCL12 plays important roles in development of chronic liver injury and a single nucleotide polymorphism (SNP) G to A change at position 801 in CXCL12 gene has been demonstrated to affect CXCL12 production levels.

Objective: This study aimed to analyze the association of CXCL12 G801A SNP with liver complication in HIV-infected Thais.

Methods: A cross-sectional study was conducted in 164 patients who were evaluated for transaminitis and significant liver fibrosis, defined by fibrosis-4 (FIB-4) score and AST to platelet ratio index (APRI), and genotyped for the SNP using tetra-primer PCR-SSP.

Results: There were high rates of patients with transaminits (28.0\%), and significant liver fibrosis by FIB-4 score (18.9\%) and by APRI (14.0\%). The CXCL12 G801A AA/GA genotypes were significantly associated with transaminitis $(p=0.014)$ and significant fibrosis by APRI $(p=0.020)$. Univariate and multivariate analyses identified the AA/GA genotypes as predictive factors for significant fibrosis (OR 6.8, 95\%CI 1.7-28.2, $p=0.008$ ), together with age older than 40 years, $\mathrm{CD}^{+}$cell count $<350$ cells/ $\mu$ and hepatitis B and/or C virus coinfection. The significantly higher medians of APRI and FIB-4 score, in patients with AA/GA than those with GG genotypes $(p<0.05)$ were observed in the ART-naïve, but not ART-experienced groups.
\end{abstract}

Conclusion: The CXCL12 G801A AA/GA genotypes are significant predictive factors for hepatic fibrosis potentially in the ART-naïve HIV-infected Thais.

Key words: CXCL12 G801A polymorphism, HIV, liver fibrosis, APRI, FIB-4

\footnotetext{
From:

${ }^{1}$ Graduate Program in Medical Technology,

${ }^{2}$ Department of Medical Technology,

${ }^{3}$ Department of Physical Therapy, Faculty of Allied Health Sciences,

Thammasat University, Pathumthani, Thailand

${ }^{4}$ Bureau of AIDS, TB and STIs, Department of Disease Control,

Ministry of Public Health, Tiwanon Road, Muang District,

Nonthaburi, Thailand

Nakorn Nayok Hospital, Nakorn Nayok, Thailand
}

\section{Background}

Human immunodeficiency virus (HIV) infection continues to be a major heath issue with estimated 36.7 million people living with HIV worldwide at the end of 2015. ${ }^{1}$ During the last decade, the advances in management of HIV-infected patients with combined anti-retroviral therapy (ART) have led to a decrease of acquired immunodeficiency syndrome (AIDs)-related morbidity and mortality with an increased

\author{
Corresponding author: \\ Chareeporn Akekawatchai \\ Department of Medical Technology, Faculty of Allied Health Sciences, \\ Thammasat University, 99 Moo 18, Klongluang, Pathumthani, \\ Thailand \\ E-mail: cakekawatchai@gmail.com, ejareepo@tu.ac.th
}

evidence of liver-related diseases. Various factors can cause liver damage in HIV-infected patients including HIV replication in the liver, hepatitis $\mathrm{B}$ or $\mathrm{C}$ virus (HBV or $\mathrm{HCV}$ ) coinfection, anti-retroviral therapy (ART), immune reconstitution and opportunistic infections. ${ }^{2,3}$ Viral hepatitis has been shown to be the most common risk of liver-related illness in HIV patients. ${ }^{2-4}$ Published studies in HIV-monoinfected and hepatitis 
virus-coinfected patients have also identified multiple risk factors for development and severity of liver disease including HIV RNA levels, $\mathrm{CD}^{+}$cell count, increased body mass index, duration and types of ART, severe alcohol use, diabetes mellitus and host genetic polymorphisms. ${ }^{5-8}$ Presently, even though studies have revealed some genetic and immunological parameters influencing liver disease progression specifically in $\mathrm{HCV} / \mathrm{HIV}$-coinfected patients, ${ }^{8}$ host genetic determinants of liver disease in HIV infection are required more investigation.

Chemokine systems play important roles in the recruitment of immune cells to the liver, regulating immune cell infiltration, chronic inflammation and progression of fibrosis. ${ }^{9}$ One of the most interesting systems is CXCL12 or stromal cell -derived factor-1 (SDF-1) and its respective receptors CXCR4 and CXCR7. The CXCL12/CXCR4/CXCR7 system is known to regulate liver homeostasis, and their increased expression are involved in acute liver injury, chronic liver damage or fibrosis, and hepatocellular carcinoma (HCC) development and metastasis. ${ }^{9,10}$ Apparently, the common polymorphism in CXCL12 gene, a single nucleotide polymorphism (SNP) G to A change at position 801 in the 3'untranslated region (UTR) of an alternatively spliced mRNA transcript, also termed SDF13'A or rs1801157, has been reported to affect levels of CXCL12 production in vitro and in vivo. ${ }^{11-13} \mathrm{Up}$ to date, only few studies have investigated roles of CXCL12 gene polymorphisms in liver diseases. SNPs in CXCL12 gene have been demonstrated to influence occult $\mathrm{HBV}$ infection, therapeutic responses in $\mathrm{HCV} / \mathrm{HIV}$ coinfection, and susceptibility and pathological development of hepatocellular carcinoma (HCC), ${ }^{14-16}$ but lack of significant association with death and HCC occurrence in HCV-related and alcoholic cirrhosis. ${ }^{17,18}$ CXCL12 is also well known to contribute dramatically to pathogenesis and progression of HIV infection and many previous studies have indicated an impact of CXCL12 gene polymorphisms including the G801A SNP on resistance of HIV infection, progression to AIDs and responses to ART. ${ }^{11,13,19-22}$ However, influence of the CXCL12 gene polymorphism on chronic liver diseases developed in HIV-infected patients is still unknown.

Our previous study has demonstrated a high prevalence of transaminitis in Thai HIV-infected patient group with high rates of $\mathrm{HBV}$ and/or $\mathrm{HCV}$ coinfection and mostly on long -term suppressive ART, suggesting their potential to develop chronic liver injury. ${ }^{7}$ The present study therefore aimed to investigate an impact of CXCL12 G801A polymorphisms on hepatic complications of these patients. Frequencies of CXCL12 G801A alleles and genotypes, and their association with liver abnormalities were examined. Risk factors for liver complications in this HIV patient group were also determined using logistic regression analyses.

\section{Materials and Methods Study population}

A cross-sectional study was conducted in $164 \mathrm{HIV}$ patients attending the Antiretroviral Therapy Clinic in Nakorn Nayok Hospital from October 2011 to June 2013. In this study, inclusion criteria were as follows: patients with documented HIV infection, and availability of blood samples and clinical data. Patients who regularly consumed alcohol, herbal medicine, steroid medication, and patients with active opportunistic infections including tuberculosis, were excluded. ${ }^{2,23}$ All subjects provided written informed consent and the study protocol was reviewed and approved by the Human Ethics Committees No. 2, Thammasat University, Thailand (Project no. 077/256).

Clinical and laboratory data were obtained as described in the previous study. ${ }^{7}$ Briefly, the data collected from patient's medical records and standardized questionnaire were age, gender, duration of antiretroviral therapy (ART), currently used anti-retroviral (ARV) regimens, opportunistic infections, alcohol consumption, herbal medicine and steroid intake. All patients underwent clinical examination and routine blood tests including levels of aspartate and alanine aminotransferases (AST and ALT), platelet count, anti-HCV, HBsAg, and CD4 ${ }^{+}$cell count. Additionally, ethylene-diamine-tetra-acetic acid (EDTA) blood samples left over from routine testing were subjected to plasma separation within 8 hours after blood collection and stored at $-80^{\circ} \mathrm{C}$ until use.

\section{Genotyping of CXCL12 G801A polymorphism}

Genomic DNA was extracted from EDTA blood samples using a Nucleospin ${ }^{\circledast}$ blood extraction kit (Macherey-Nagel $\mathrm{GmbH}$, Duren, Germany) according to the manufacturer's instruction. DNA samples were genotyped for CXCL12 G801A or SDF1-3'A (rs1801157) polymorphism using a polymerase chain reaction-sequence specific primer (PCR-SSP) assay developed in the previous studies. ${ }^{24,25}$ Two pairs of specific primer sets were used. Firstly, an A allele-specific primer pair comprises forward inner (5' CATCCACATGGGAGCCA 3') and reverse outer primers (5’ ACATTGGTCACAGAGGAGGA 3') producing a 364 bp PCR product. Secondly, a G allele-specific set is composed of forward outer (5' GCTCTGAAACCAGTGTTAGG 3') and reverse inner primers (5' CCAGAAGAGGCAGACCC 3') producing a 495 bp PCR product. The two sets of primers also amplify a PCR product of $826 \mathrm{bp}$ from the two outer primers. DNA samples (25-50 $\eta$ g) were amplified using the PCR kit buffer plus $0.25 \mathrm{U}$ of Tag DNA polymerase (NanoHelix, Deajeon, South Korea). PCR conditions were as follows: denaturation at $94^{\circ} \mathrm{C}$ for $2 \mathrm{~min}, 35 \mathrm{cycles}$ of $95^{\circ} \mathrm{C}$ for $20 \mathrm{sec}$, $64.5^{\circ} \mathrm{C}$ for $50 \mathrm{sec}$, and $72^{\circ} \mathrm{C}$ for $1 \mathrm{~min}$, and elongation at $72^{\circ} \mathrm{C}$ for $5 \mathrm{~min}$ in a T100 Thermal Cycler (Bio-Rad, Hercules, CA). PCR products were then analyzed by electrophoresis in $2 \%$ agarose gel with DNA size marker of 100-1,000 bp and visualized using UV fluorescence after staining with ethidium bromide. Positive DNA controls with possible genotypes and negative control with no DNA were included in a panel of genotyping. ${ }^{24}$ As shown in Figure 1, the GG and AA genotypes yielded 495 and 364 bp PCR products respectively while the GA heterozygous genotype presented both 495 and 364 bp products. Samples of all genotypes exhibited the $826 \mathrm{bp}$ internal control fragments. Thirty four (34) of 164 PCR samples (20.7\%) with 8/164 AA, 11/164 GA and 15/164 GG genotypes were subjected to direct DNA sequencing and analyzed by Unipro UGENE v.1.24.2. There was $100 \%$ agreement between the results obtained by PCR-SSP and by direct sequencing.

\section{Evaluation of liver complication}

From routine laboratory data, several surrogate markers were used to determine liver abnormality in HIV patients including thrombocytopenia, transaminitis, fibrosis-4 (FIB-4) 


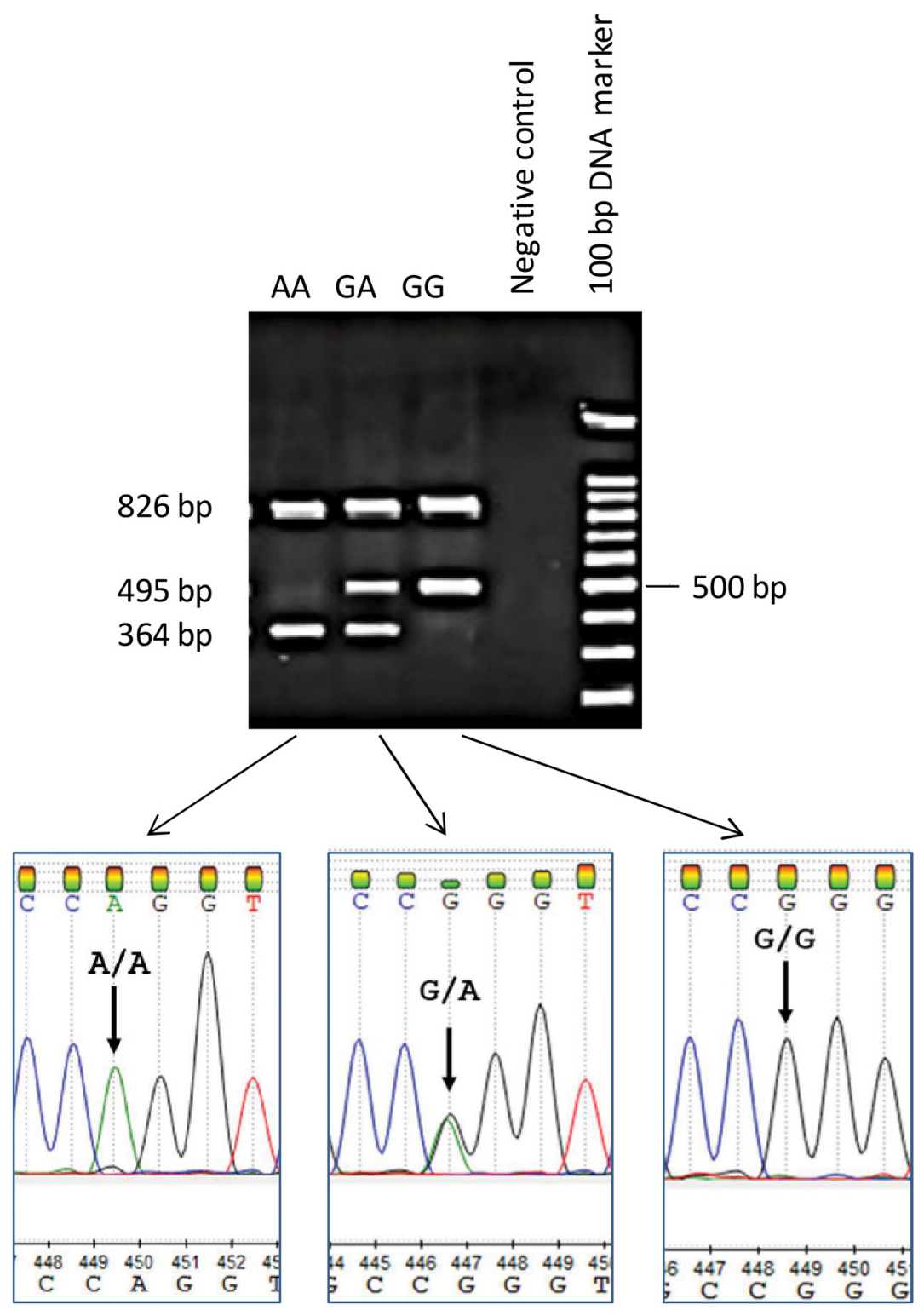

Figure 1. Genotyping of CXCL12 G801A polymorphism by tetra-primer PCR-SSP. A representative gel demonstrating AA in lane 1, GA in lane 2, GG genotypes in lane 3, negative control in lane 4 and 100 bp DNA ladders in lane 5 . The genotyping was confirmed by direct sequencing and Unipro UGENE v.1.24.2. analysis.

score and AST-to-platelet ratio index (APRI). Platelet count was utilized to assess thrombocytopenia which was classified into 4 grades. 75,000 to $150,000 / \mu \mathrm{L}$ were defined as grade $1,50,000$ to $<75,000 / \mu \mathrm{L}$ as grade $2,25,000$ to $<50,000 / \mu \mathrm{L}$ as grade 3 and $<25,000 / \mu \mathrm{L}$ as grade $4 .^{26,27}$ Transaminitis was defined as an increased level from the normal upper limit (ULN) of either AST or ALT (> $40 \mathrm{U} / \mathrm{L}){ }^{7}$ FIB-4 score was calculated and classified into class $1(<1.45)$, class $2(1.46$ to 3.25$)$ and class 3 $(>3.25) .{ }^{28,29}$ APRI value was calculated and classified into class 1 $(<0.5)$, class $2(0.51$ to 1.5$)$ and class $3(>1.5){ }^{30}$

\section{Statistical Analysis}

Genotype and allele frequencies were calculated by direct counting. Hardy-Weinberg equilibrium was assessed using $\chi^{2}$ test with one degree of freedom from online analysis tools, considering equilibrium when $p>0.05 .{ }^{31} \chi^{2}$ test or Fishers's exact test, and odds ratio with $95 \%$ confidence interval (CI) were used to determine an association of CXCL12 G801A genotypes with categorical variables. The level of statistical significance was determined at $p<0.05$. Univariate and multivariate logistic regression were performed to determine risks for the liver complication assessed by transaminitis and APRI $>0.5$. Odds ratio (OR) with $95 \%$ confidence interval (CI) and $P$ value were calculated. All $P$ values $<0.05$ were considered as statistically significant. The PASW statistics 18 software (SPSS Inc.) was used for statistical analysis.

\section{Results \\ Characteristics of the study population}

Characteristics and clinical features of the subjects are summarized in Table 1. A total of 164 patients, 90 male (54.9\%) and $74(45.1 \%)$ female with the mean age of $39.8( \pm 11.3)$ years, were included in this study. The median $\mathrm{CD} 4^{+}$cell count of these patients was 371 [1-1601] cells/ $\mu$ l. Prevalence of HBV/HIV, 
$\mathrm{HCV} / \mathrm{HIV}$ and $\mathrm{HBV} / \mathrm{HCV} / \mathrm{HIV}$ triple coinfection were $9.1 \%$ $(12 / 132), 8.3 \%(11 / 132)$ and $0.8 \%(1 / 132)$, respectively. One hundred and seventeen out of 164 patients (71.3\%) were on combined anti-retroviral (ARV) drugs with the median duration of 39 months [range, 16-55] and 104 of the 117 patients (88.9\%) were on ART for longer than 6 months. With regards to the 117 ART-experienced patients, 74 (63.2\%) were on the hepatotoxic nevirapine-based regimen.

HIV-infected patients were evaluated for liver complication using available noninvasive markers as shown in Table 1 . In the $147 \mathrm{HIV}$ patients with a mean platelet count of $276( \pm 105) \times$ $10^{9} / \mu \mathrm{L}, 10(6.8 \%)$ patients were assessed as having grade 1 or 2 thrombocytopenia. Of the 143 patients, 40 (28.0\%) had transaminitis, $20(14.0 \%)$ were assessed as having class 2 or 3 APRI $($ APRI $>0.5)$ and $27(18.9 \%)$ were classified as class 2 or 3 (FIB-4 score $>1.45)$.

Table 1. Characteristics and clinical features of Thai HIV-infected patients $(n=164)$.

\begin{tabular}{|c|c|}
\hline Characteristics & Patients N (\%) \\
\hline Patients & $164(100 \%)$ \\
\hline Age $^{\mathrm{a}}$ (years) & $39.8( \pm 11.3)$ \\
\hline Gender (male) & $90(54.9 \%)$ \\
\hline $\mathrm{CD}^{+}$cell count $($cells/ $\mu \mathrm{l})(\mathrm{n}=141)$ & $371[1-1601]$ \\
\hline $\begin{array}{l}\text { HBV or HCV coinfection }(\mathrm{n}=132) \\
\text { HIV monoinfection } \\
\text { HIV/HBV coinfection } \\
\text { HIV/HCV coinfection } \\
\text { HIV/HBV/HCV triple-infection }\end{array}$ & $\begin{array}{l}108(81.8 \%) \\
12(9.1 \%) \\
11(8.3 \%) \\
1(0.8 \%)\end{array}$ \\
\hline $\begin{array}{l}\text { Current ARV regimens } \\
\text { Naive to ARV treatment } \\
\text { Lamivudine/Stavudine/Nevirapine } \\
\text { Lamivudine/Zidovudine/Nevirapine } \\
\text { Lopinavir/Ritonavir or Atazanavir } \\
\text { Others }\end{array}$ & $\begin{array}{l}47(28.7 \%) \\
11(6.7 \%) \\
57(34.8 \%) \\
10(6.1 \%) \\
39(23.8 \%)\end{array}$ \\
\hline $\begin{array}{l}\text { Nevirapine experience }(\mathrm{n}=117) \\
\text { Nevirapine-based regimens }\end{array}$ & $74(63.2 \%)$ \\
\hline Duration of ARV treatment $(\mathrm{n}=117)^{\mathrm{b}}$ & $39[16-55]$ \\
\hline $\begin{array}{l}\text { Duration of ARV treatment }(\mathrm{n}=117) \\
\leq 6 \text { months } \\
>6 \text { months }\end{array}$ & $\begin{array}{l}13(11.1 \%) \\
104(88.9 \%)\end{array}$ \\
\hline $\begin{array}{l}\text { Platelet count }{ }^{\mathrm{a}}(\mathrm{n}=147) \\
\quad \text { Platelet count }\left(\times 10^{9} / \mathrm{L}\right)\end{array}$ & $276( \pm 105)$ \\
\hline $\begin{array}{l}\text { Thrombocytopenia }(\mathrm{n}=147) \\
\text { Grade } 1 \text { or } 2(75,000-150,000 \text { or } 50,000-<75,000 / \mu \mathrm{l})\end{array}$ & $10(6.8 \%)$ \\
\hline $\begin{array}{l}\text { Transaminitis }(\mathrm{n}=143) \\
\text { AST and/or ALT }>\text { ULN }(40 \mathrm{U} / \mathrm{L})\end{array}$ & $40(28.0 \%)$ \\
\hline $\begin{array}{l}\text { Significant liver fibrosis }(\mathrm{n}=143) \\
\text { FIB- } 4 \text { score }>1.45 \\
\text { APRI }>0.5\end{array}$ & $\begin{array}{l}27(18.9 \%) \\
20(14.0 \%)\end{array}$ \\
\hline
\end{tabular}

${ }^{\mathrm{a}}$ and ${ }^{\mathrm{b}}$ Data shown as mean value \pm S.D and, median and interquartile range [IQR] respectively. Some variables had missing data and $\mathrm{n}$ is given in parentheses. AST: aspartate aminotransferase, ALT: alanine aminotransferase, FIB-4: fibrosis-4 score, APRI: AST to platelet ratio index
Genotypic and allelic distribution of CXCL12 G801A polymorphism and association with liver complication in HIV-infected Thais

As shown in Table 2, 93 (56.7\%), 58 (35.4\%) and 13 (7.9\%) patients were detected with GG, GA and AA genotypes and frequencies of $\mathrm{G}$ and $\mathrm{A}$ alleles were 0.744 (244) and 0.256 (84) respectively. In the dominant model, the percentages of GG and combined GA/AA genotypes were 56.7\% (93/164) and $43.3 \%$ (71/164) consequently. The CXCL12 genotypes were consistent with the Hardy-Weinberg equilibrium $(p>0.05)$. Statistic analysis indicated that the characteristics of the patients possessing AA, GA and GG genotypes, and A and G alleles, including $\mathrm{CD}^{+}$cell count, HBV and HCV coinfection, and FIB-4 score were similar $(p>0.05)$, except for transaminitis $(p=0.024$, and $p=0.006$ respectively) and fibrosis assessed by having APRI > 0.05 ( $p=0.007$ and $p=0.003$ respectively). Genotypes under the dominant model were also significantly associated with transaminitis (OR 2.5, 95\%CI 1.2-5.3, $p=0.014$ ) and having APRI $>0.5$ (OR 3.1, 95\%CI 1.2-8.4, $p=0.020$ ). Notably, there was no statistically significant difference between the patient groups with different genotypes and alleles regarding ARV regimens currently used, nevirapine experience and duration of ART ( $p>$ 0.05) (data not shown).

\section{CXCL12 G801A polymorphism as a predictive factor for liver complication in the ART-naïve patients}

To examine possible risk factors for liver abnormalities assessed by transaminitis and APRI in this study population, logistic regression analyses were conducted on variables which were CXCL12 G801A genotypes, gender, age, CD4+ cell count, hepatitis $\mathrm{B}$ and/or $\mathrm{C}$ virus coinfection, navirapine experience and ART duration as shown in Table 3. Univariate analysis of risk factors for transaminitis indicated that being male, coinfection with HBV and/or HCV and the CXCL12 G801A AA/ GA genotypes were risk factors for developing transaminitis (OR 3.7, 95\%CI 1.6-8.4, $p=0.002$, OR 3.3, 95\%CI 1.3-8.9, $p=$ 0.015 and $\mathrm{OR} 2.5,95 \% \mathrm{CI} 1.2-5.3, p=0.015$, respectively). In subsequent multivariate analysis, only coinfection with $\mathrm{HBV}$ and/or HCV was predictive for transaminitis in this study group (OR 3.1, 95\%CI 1.1-8.6, $p=0.035$ ). In univariate analysis of risk factors for having APRI $>0.5$, several factors including being male, age older than 40 years, having $\mathrm{CD} 4^{+}$cell count $<350$ cells/ $\mu$ l, coinfection with $\mathrm{HBV}$ and/or HCV and the CXCL12 G801A AA/GA genotypes were identified as predictors of having APRI $>0.5$ (OR 3.6, 95\%CI 1.1-11.2, $p=0.030$, OR 3.1, 95\%CI 1.1-8.6, $p=0.031$, OR 5.3, 95\%CI 1.7-17.0, $p=0.004$, OR 4.2, 95\%CI 1.4-12.7, $p=0.013$, OR 3.1, 95\%CI $1.2-8.4, p=0.025$, respectively). Multivariate analysis subsequently indicated that the AA/GA genotypes together with age older than 40 years, $\mathrm{CD}^{+}$cell count $<350$ cells/ $\mu$ l and HBV and/or HCV coinfection remained predictive factors of having APRI $>0.5$ in this study population (OR 6.8, 95\%CI 1.7-28.2, $p$ $=0.008$, OR 7.5, 95\%CI 1.7-34.4, $p=0.009$, OR 7.7, 95\%CI 1.5$38.5, p=0.012$, OR 6.5, 95\%CI 1.5-27.4, $p=0.010$, respectively). The analysis did not show significant association of both transaminits and having APRI $>0.5$ with nevirapine experience and ART duration $(p>0.05)$. 


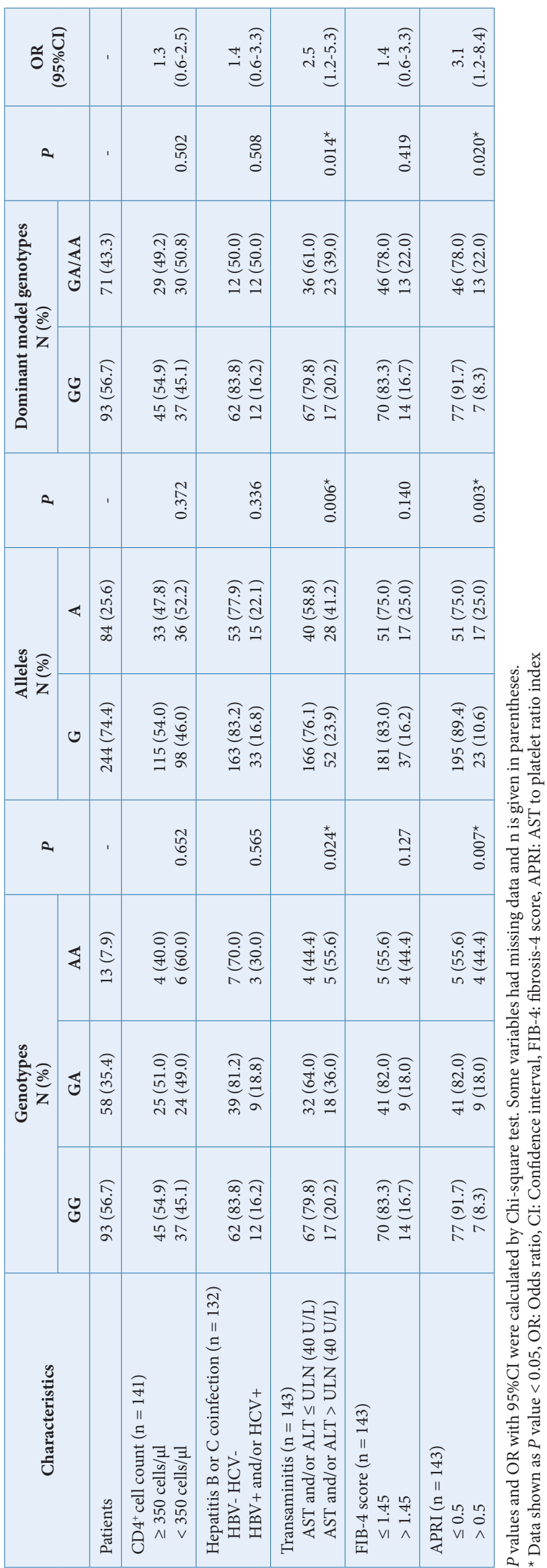

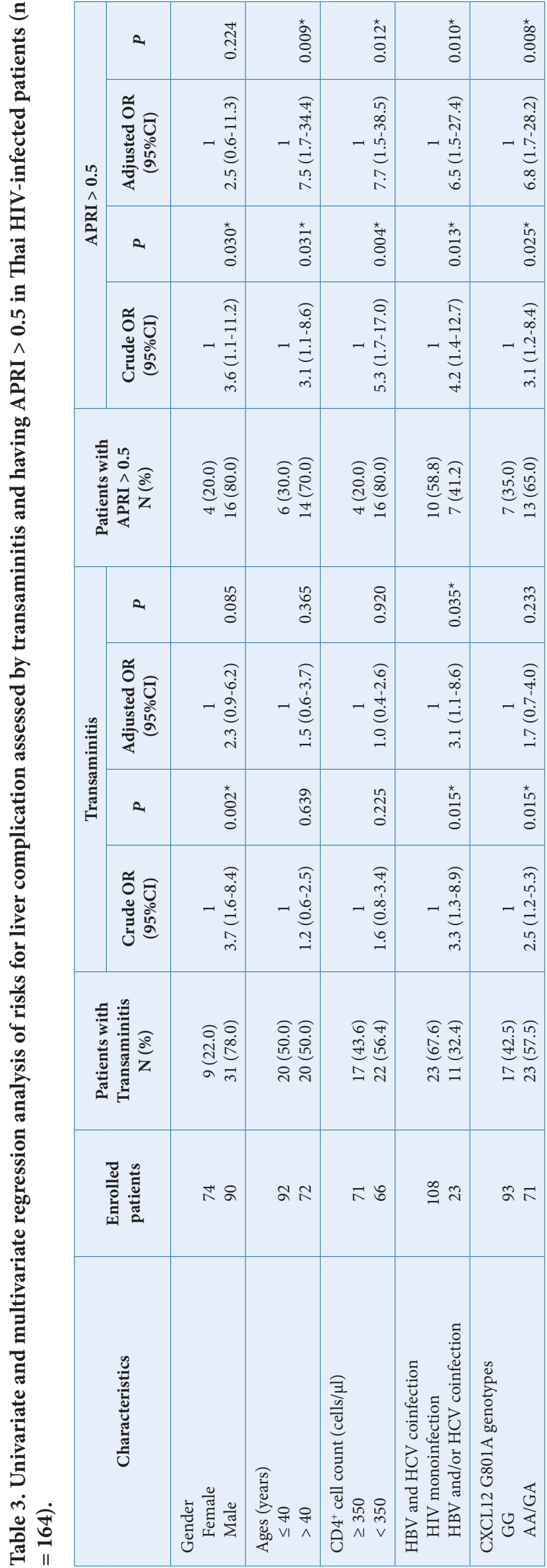



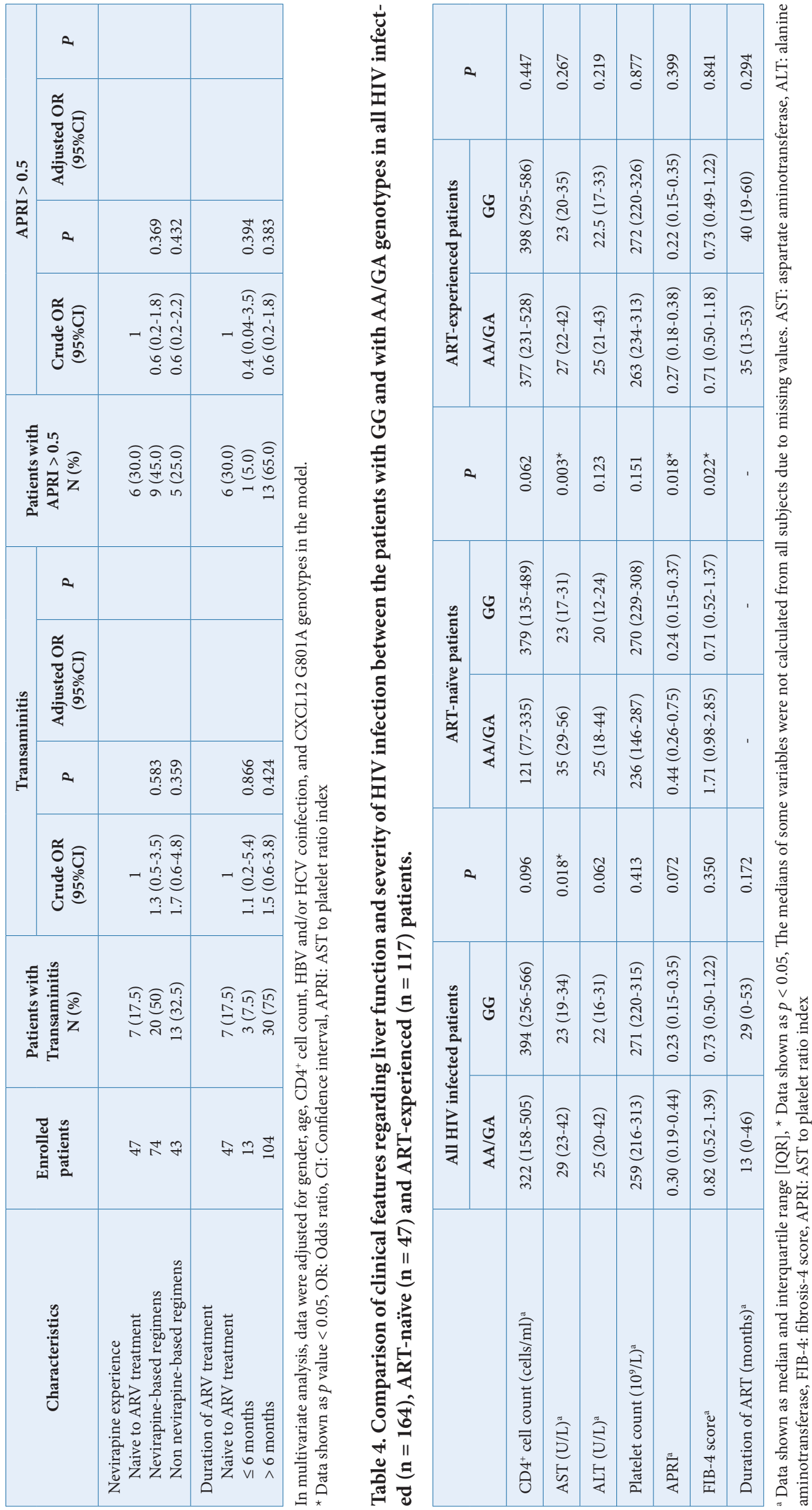

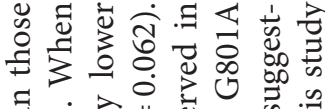

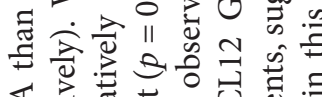

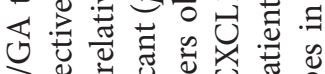

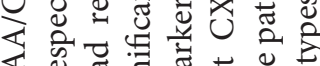

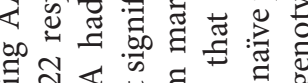

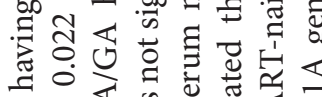

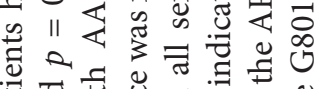

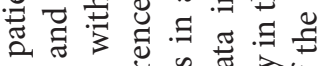

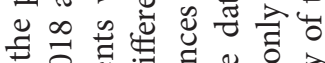

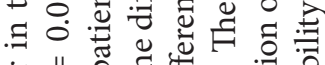

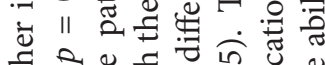
总

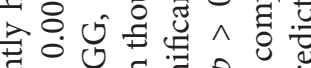

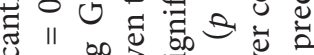
는

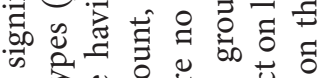

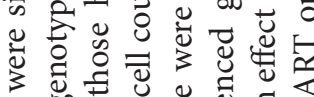

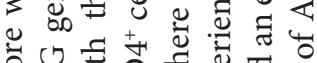

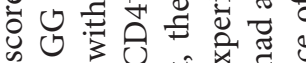

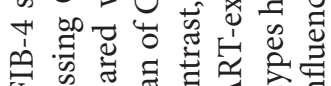

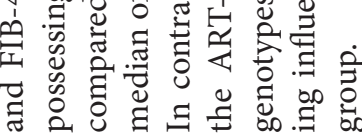

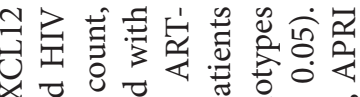

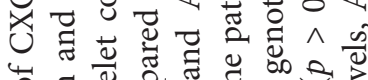

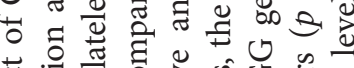

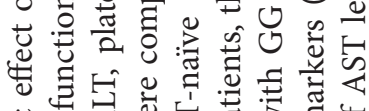

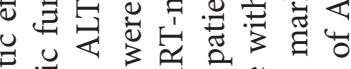

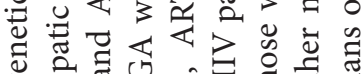

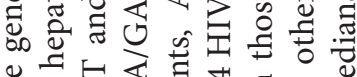

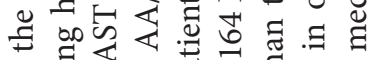

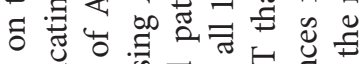

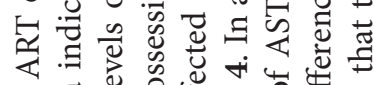

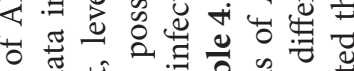

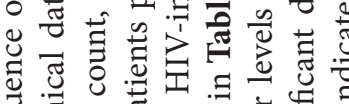

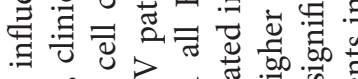

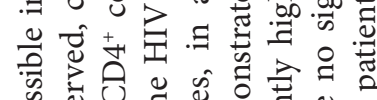

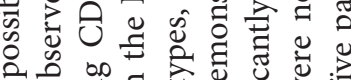

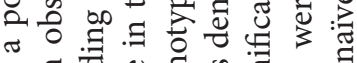

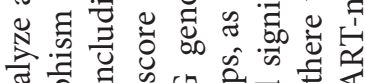

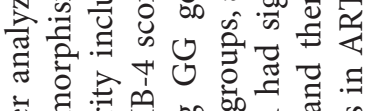

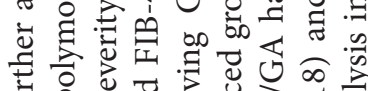

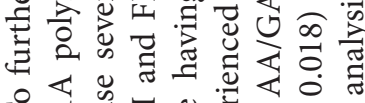

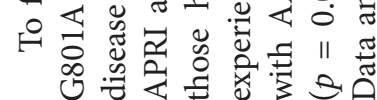




\section{Discussion}

This cross-sectional study was conducted in 164 HIV patients maintaining high $\mathrm{CD}^{+}$cell count. There were high prevalence rates of $\mathrm{HBV}$ and $\mathrm{HCV}$ coinfection and high percentages of patients with long suppressive ART especially hepatotoxic nevirapine-containing regimens. Evaluation of liver abnormalities by non-invasive laboratory test available in this study indicated that there were high rates of patients with thrombocytopenia (6.8\%), transaminitis $(28.0 \%)$, significant fibrosis as shown by FIB-4 score $>1.45(18.9 \%)$ and by APRI $>$ $0.5(14.0 \%)$ consistent with previous studies reporting prevalence of liver fibrosis in HIV-monoinfected and HCV-coinfected patients. ${ }^{6,32}$ Our analysis indicated a high prevalence of HIV patients with chronic hepatic injury, which possibly develop to more severe fibrosis and cirrhosis as observed in the studies in HIV patients who were coinfected with HCV and on ART for longer duration. ${ }^{32,33}$

CXCL12 G801A polymorphism has been well characterized in different ethnic groups and established to have an impact on susceptibility to HIV infection, HIV disease progression and ART response ${ }^{11,13,19-22}$ whereas few studies indicate its role in liver disease caused by hepatitis viruses and hepatocellular carcinoma. ${ }^{14,16}$ In this study, frequency of the A allele of CXCL12 G801A polymorphism was observed as 0.256 while previous studies in Thai HIV-seropositive patients and blood donors reported the frequencies ranging from 0.199-0.43..$^{20,21}$ The CXCL12 G801A genotypes were in Hardy-Weinberg equilibrium $(p>0.05)$, indicating that genetic background of the study population remains constant. Our data indicated significant associations of the CXCL12 G801A SNP with transaminitis and significant hepatic fibrosis assessed by APRI $>0.5$. While univariate analysis suggested the G801A AA/ AG genotypes as risk factors for transaminitis, subsequent multivatiate analysis indicated no longer significant risk of the genotypes. However, the analyses have identified the G801A AA/GA genotypes as risk factors for significant liver fibrosis by APRI and it is apparent that the patients with AA/GA carries 6.8 times higher risk than those with GG genotypes. Interestingly, additional analysis demonstrated the significant higher medians of AST, APRI and FIB-4 score, with the relatively lower $\mathrm{CD} 4^{+}$cell count, in patients possessing the AA/GA than those having GG genotypes observed only in the ART-naïve, but not in ART-experienced HIV patient groups. Notably, distribution of the G801A AA/GA and GG genotypes in the ART-naive and in ART-experienced patients are similar $(p>0.05)$ (data not shown). Collectively, the results suggest a strongly significant effect of the G801A AA/GA genotypes on liver abnormalities during HIV infection, and this effect can possibly be lessened by ARV treatment. Mechanisms for the neutralization of this genetic effect by ART are unclear and remain to be elucidated. To the best of our knowledge, this is the first evidence implicating the CXCL12 G801A polymorphism in the development of liver fibrosis in HIV-infected patients.

Functional roles of the CXCL12 G801A SNP have been studied in several diseases including HIV infection, but have not been clearly described in liver diseases. The G801A SNP is known to be located in a segment of the 3'UTR of $\beta$ isoform transcript that may serve as a target for cis-acting factors promoting synthesis of CXCL12. ${ }^{11}$ The in vitro study demonstrated that the 3'A genotype leads to an enhanced CXCL12 mRNA stability and therefore increases levels of the CXCL12 mRNA production. ${ }^{12}$ The in vivo effect of the G801A SNP on CXCL12 production has also been demonstrated in HIV infection, ${ }^{13,20}$ even though its mechanistic roles in susceptibility and disease progression are still controversial and not completely clear. ${ }^{11,13,19}$ In liver microenvironment, CXCL12 is constitutively produced mainly by biliary epithelial cells and apparently upregulated during fibrotic injury, leading to an elevation of plasma CXCL12 levels in the patients with chronic liver inflammation and fibrosis whereas over $50 \%$ of liver infiltrating cells are CXCR4 positive. ${ }^{34}$ In chronic liver injury, CXCL12 is increasingly expressed and activates CXCR4 on liver sinusoidal endothelial cells, triggering a profibrotic response, and induces hepatic stellate cells (HSC) proliferation and activation, leading to $a$-smooth muscle actin and collagen production. CXCL12 also attracts mesenchymal stem cells from the bone marrow that can promote liver fibrosis. ${ }^{10}$ Accordingly, production of CXCL12 particularly in the liver area, which is possibly released into circulation, may influence hepatic fibrogenesis and therefore affecting progression of chronic liver diseases. Thus, an influence of the CXCL12 G801A genotypes on significant fibrosis observed in our HIV-infected patients may be due to their effect on the CXCL12 expression and function during chronic liver injury.

As there were multiple factors possibly associated with liver abnormalities in this study group, logistic regression analysis for risks of liver complication was adjusted for characteristics of patients including gender, age, $\mathrm{CD}^{+}$cell count, $\mathrm{HBV}$ and/ or HCV coinfection, CXCL12 G801A genotypes, navirapine experience and ART duration in the model. Consistent with the findings in our previous study, ${ }^{7} \mathrm{HBV}$ and/or HCV coinfection remains a risk factor for transaminitis in this study group. Apart from the G801A AA/GA genotypes, other predictive factors for significant liver fibrosis in this study group including age older than 40 years, $\mathrm{CD}^{+}$cell count $<350$ cells $/ \mu \mathrm{l}$ and HBV and/or HBV coinfection were also identified. These risk factors are probably different from many previous studies due to differences in the study design, characteristics of study population, outcome variables, ARV drug regimens and duration of ART. ${ }^{2,3,5,6,35}$ However, it is important to note limitations of this study. Firstly, a cross-sectional study design is usually unable to control confounding factors although the multivariate regression analysis was applied to this association study. Besides, this study design may not allow determining the precise effect of particular ARV drugs on hepatic abnormalities because the patients possibly have been switched from other regimens. Secondly, this study contains a limited number of subjects and missing values in some variables, which may limit statistical significance for the variables tested. Thirdly, there is no functional assessment of the G801A genotypes in this study and further evaluation of CXCL12 activities both in circulation and hepatic area is suggested. Fourthly, haplotype analysis of the rs1801157, G801A SNP, along with other SNPs in the 3' UTR of CXCL12 gene such as rs1029153 should be performed to confirm its significance in the liver complication 
in HIV infection. Lastly, this effect of the G801A SNP on liver complication was analyzed only in Thai patients and it would be interesting to extend the analysis to different ethnic groups.

\section{Conclusion}

This cross-sectional SNP analysis provides the evidence that the presence of CXCL12 G801A SNP is associated with development of liver complication probably in the ARTnaïve HIV-infected Thais. Specifically, the patients having the unfavorable G801A AA/GA had a significantly higher risk for significant liver fibrosis than those carrying GG genotypes. This finding might provide a new molecular genetic mechanism for liver disease in HIV patients. A further prospective study in a larger number of patients is required to verify our finding.

\section{Acknowledgments}

This study was supported by the 2016 fiscal year budget of Thammasat University and Thitiilat Chiraunyanann is a recipient of the Research Grant for Graduate Studies from the National Research Council of Thailand and from Thammasat University (the Fiscal year 2014). Special thanks are due to all patients participated in this study.

\section{Authors'disclosures of potential conflicts of inter- est}

No conflict of interest is declared.

\section{References}

1. World Health Organization[Internet]. Geneva: World Health Organization; c2017 [cited 2017 July 1]. Media Centre: HIV/AIDS; [about 10 screens]. Available from: http://www.who.int/mediacentre/factsheets/fs360/en/.

2. Joshi D, O'Grady J, Dieterich D, Gazzard B, Agarwal K. Increasing burden of liver disease in patients with HIV infection. Lancet. 2011;377:1198-209.

3. Puri P, Kumar S. Liver involvement in human immunodeficiency virus infection. Indian J Gastroenterol. 2016;35:260-73.

4. Smith C, Sabin CA, Lundgren JD, Thiebaut R, Weber R, Law M,et al. Data Collection on Adverse Events of Anti H.I.V. drugs study group. Factors associated with specific causes of death amongst HIV-positive individuals in the D:A:D Study. AIDS. 2010;24:1537-48.

5. Kovari H, Ledergerber B, Battegay M, Rauch A, Hirschel B, Foguena A $\mathrm{K}$, et al. Incidence and risk factors for chronic elevation of alanine aminotransferase levels in HIV-infected persons without hepatitis b or c virus co-infection. Clin Infect Dis. 2010;50:502-11.

6. DallaPiazza M, Amorosa VK, Localio R, Kostman JR, Lo Re V 3rd. Prevalence and risk factors for significant liver fibrosis among HIV -monoinfected patients. BMC Infect Dis. 2010;10:116.

7. Akekawatchai C, Sretapunya W, Pipatsatitpong D, Chuenchit T. Hepatitis $\mathrm{B}$ or $\mathrm{C}$ virus coinfection in and risks for transaminitis in human immunodeficiency virus - infected Thais on combined antiretroviral therapy. Asian Biomed (Res Rev News). 2015;9:353-61.

8. Medrano LM, Jimenez-Sousa MA, Fernandez-Rodriguez A, Resino S. Genetic Polymorphisms Associated with Liver Disease Progression in HIV/HCV-Coinfected Patients. AIDS Rev. 2017;19:3-15.

9. Saiman Y, Friedman SL. The role of chemokines in acute liver injury. Front Physiol. 2012;3:213.

10. Liepelt A, Tacke F. Stromal cell-derived factor-1 (SDF-1) as a target in liver diseases. Am J Physiol Gastrointest Liver Physiol. 2016;311:G203-9.

11. Winkler C, Modi W, Smith MW, Nelson GW, Wu X, Carrington M, et al. Genetic restriction of AIDS pathogenesis by an SDF-1 chemokine gene variant. ALIVE Study, Hemophilia Growth and Development Study (HGDS), Multicenter AIDS Cohort Study (MACS), Multicenter Hemophilia Cohort Study (MHCS), San Francisco City Cohort (SFCC). Science. 1998;279:389-93.
12. Garcia-Moruja C, Rueda P, Torres C, Alcami J, Luque F, Caruz A. Molecular phenotype of CXCL12beta 3'UTR G801A polymorphism (rs1801157) associated to HIV-1 disease progression. Curr HIV Res. 2009;7:384-9.

13. Soriano A, Martinez C, Garcia F, Plana M, Palou E, Lejeune M, et al. Plasma stromal cell-derived factor (SDF)-1 levels, SDF1-3'A genotype, and expression of CXCR4 on T lymphocytes: their impact on resistance to human immunodeficiency virus type 1 infection and its progression. J Infect Dis. 2002;186:922-31.

14. Hassanshahi G, Arababadi MK, Khoramdelazad H, Yaghini N, Zarandi ER. Assessment of CXCL12 (SDF-1alpha) polymorphisms and its serum level in posttransfusion occult HBV-infected patients in Southeastern Iran. Arch Med Res. 2010;41:338-42.

15. Pineda-Tenor D, Jimenez-Sousa MA, Rallon N,Berenguer J, Soriano V, Aldamiz-Echevarria T,et al. Short Communication: CXCL12 rs1029153 Polymorphism Is Associated with the Sustained Virological Response in HIV/Hepatitis C Virus-Coinfected Patients on Hepatitis C Virus Therapy. AIDS Res Hum Retroviruses. 2016;32:226-31.

16. Chang CC, Chen SC, Hsieh YH, Chen YC, Chen T Y, Chu YH,et al. Stromal cell-derived factor-1 but not its receptor, CXCR4, gene variants increase susceptibility and pathological development of hepatocellular carcinoma. Clin Chem Lab Med. 2009;47:412-8.

17. Nahon P, Sutton A, Rufat P,Faisant C, Simon C, Barget N, et al. Lack of association of some chemokine system polymorphisms with the risks of death and hepatocellular carcinoma occurrence in patients with alcoholic cirrhosis: a prospective study. Eur J Gastroenterol Hepatol. 2007;19:425-31.

18. Nahon P, Sutton A, Rufat P, Simon C, Trinchet J C, Gattegno L, et al. Chemokine system polymorphisms, survival and hepatocellular carcinoma occurrence in patients with hepatitis C virus-related cirrhosis. World J Gastroenterolc. 2008;14:713-9.

19. Mummidi S, Ahuja SS, Gonzalez E, Anderson S A, Santiago E N, Stephan K.T,et al. Genealogy of the CCR5 locus and chemokine system gene variants associated with altered rates of HIV-1 disease progression. Nat Med. 1998;4:786-93.

20. Tiensiwakul P. Stromal cell-derived factor (SDF) 1-3'A polymorphism may play a role in resistance to HIV-1 infection in seronegative high-risk Thais. Intervirology. 2004;47:87-92.

21. Ammaranond P, Sanguansitthianan S, Phaengchomduan P, Sae-Lee C, Mardkhumchan S. Impact of CCR2 and SDF1 polymorphisms on disease progression in HIV-infected subjects in Thailand. J Clin Lab Anal. 2013;27:38-44

22. Puissant B, Roubinet F, Massip P, Sandres-Saune K, Apoil PA, Abbal M, et al. Analysis of CCR5, CCR2, CX3CR1, and SDF1 polymorphisms in HIV-positive treated patients: impact on response to HAART and on peripheral T lymphocyte counts. AIDS Res Hum Retroviruses. 2006; 22:153-62.

23. Lomtadze N, Kupreishvili L, Salakaia A, Vashakidze S, Sharvadze L, Kempker, RR,et al. Hepatitis C virus co-infection increases the risk of anti-tuberculosis drug-induced hepatotoxicity among patients with pulmonary tuberculosis. PLoS One. 2013;8:e83892.

24. Kingkeow D. The influence of cytokine, chemokine and chemokine receptor gene polymorphisms and their expression on HIV disease progression. [dissertation] Chiang Mai: Chiang Mai University; 2009.207 p.

25. Ye S, Dhillon S, Ke X, Collins AR, Day IN. An efficient procedure for genotyping single nucleotide polymorphisms. Nucleic Acids Res. 2001; 29:E88-8.

26. Sekhon SS, Roy V. Thrombocytopenia in adults: A practical approach to evaluation and management. South Med J. 2006;99:491-8; quiz 9-500, 33.

27. Afdhal N, McHutchison J, Brown R, Jacobson I, Manns M, Poordad F, et al. Thrombocytopenia associated with chronic liver disease. J Hepatol. 2008;48:1000-7.

28. Sterling RK, Lissen E, Clumeck N, Sola, R, Correa MC, Montaner J, et al. Development of a simple noninvasive index to predict significant fibrosis in patients with HIV/HCV coinfection. Hepatology. 2006;43:1317-25.

29. Foca E, Fabbiani M, Prosperi M, Quiros Roldan E, Castelli F, Maggiolo F, et al. Liver fibrosis progression and clinical outcomes are intertwined: role of CD4+ T-cell count and NRTI exposure from a large cohort of HIV/ HCV-coinfected patients with detectable HCV-RNA: A MASTER cohort study. Medicine (Baltimore). 2016;95:e4091.

30. Wai CT, Greenson JK, Fontana RJ,Kalbfleisch JD, Marrero JA, Conjeevaram HS, et al. A simple noninvasive index can predict both significant fibrosis and cirrhosis in patients with chronic hepatitis C. Hepatology. 2003; 38:518-26. 
31. Rodriguez S, Gaunt TR, Day IN. Hardy-Weinberg equilibrium testing of biological ascertainment for Mendelian randomization studies. Am J Epidemiol. 2009;169:505-14.

32. Pineda-Tenor D, Berenguer J, Garcia-Alvarez M, Guzman-Fulgencio M, Carrero A, Aldamiz-Echevarria T, et al. Single nucleotide polymorphisms of CXCL9-11 chemokines are associated with liver fibrosis in HIV/ HCV-coinfected patients. J Acquir Immune Defic Syndr. 2015;68:386-95.

33. Guzman-Fulgencio M, Berenguer J, Jimenez-Sousa MA,Pineda-Tenor D, Aldamiz-Echevarria T, Garcia-Broncano P, et al.Association between IL7R polymorphisms and severe liver disease in HIV/HCV coinfected patients: a cross-sectional study. J Transl Med. 2015;13:206.
34. Wald O, Pappo O, Safadi R, Dagan-Berger M, Beider K,Wald H, et al. Involvement of the CXCL12/CXCR4 pathway in the advanced liver disease that is associated with hepatitis $\mathrm{C}$ virus or hepatitis $\mathrm{B}$ virus. Eur J Immunol. 2004;34:1164-74

35. Chalermchai T, Hiransuthikul N, Tangkijvanich P, Pinyakorn $S$, Avihingsanon A, Ananworanich J. Risk factors of chronic hepatitis in antiretroviral-treated HIV infection, without hepatitis B or C viral infection. AIDS Res Ther. 2013;10:21. 